\title{
Caracterizando a Pesquisa em Informática na Educação no Brasil: Um Mapeamento Sistemático das Publicações do SBIE
}

\author{
Cleyton V. C. Magalhães ${ }^{1}$, Ronnie E. S. Santos ${ }^{1}$, Fabio Q. B. da Silva ${ }^{1}$, Alex Sandro \\ Gomes ${ }^{1}$ \\ ${ }^{1}$ Centro de Informatica (CIn) \\ Universidade Federal de Pernambuco (UFPE) \\ Cidade Universitária, 50.740-560 Recife, PE, Brasil \\ \{ress, cvcm, fabio, asg\}@cin.ufpe.br
}

\begin{abstract}
Context: The Brazilian Symposium on Computer in Education (SBIE) has established itself as the main scientific event in this area in Brazil. It is considered as an important conference to disseminate results, discuss trends and to promote interaction among researchers. Goal: This paper aims to plot the landscape of computer in education area in Brazil, based on papers published on SBIE between 2001 and 2012. Method: The method used in this paper is a mapping study, which is a secondary research applied to integrate results gathered from several studies published previously. Results and Conclusion: The results show that the research in computer in education in Brazil is diverse with respect to the researched topics, and various contexts are covered. However, it is restricted regarding the research methods. It can also be highlighted the low occurrence of empirical methods.
\end{abstract}

Resumo. Contexto: O Simpósio Brasileiro de Informática na Educação (SBIE) se consolidou como o principal evento científico da área no Brasil, sendo para pesquisadores e educadores, um importante ambiente de divulgação de resultados, discussão de tendências e interação entre grupos que pesquisam o tema. Objetivo: Este trabalho tem o objetivo verificar como se caracteriza a pesquisa em informática na educação no Brasil com base nas publicações do SBIE entre os anos de 2001 e 2012. Método: O método utilizado nesta pesquisa é um mapeamento sistemático, método de pesquisa secundário empregado para integrar os resultados oriundos de diversos estudos publicados anteriormente. Resultados e Conclusão: Os resultados mostram que a pesquisa em informática na educação no país é diversificada em relação aos tópicos pesquisados, abrangendo diversos contextos, porém é limitada em relação aos métodos de pesquisa, apresentado um número relativamente baixo de pesquisas empíricas.

\section{Introdução}

A História da Informática na Educação no Brasil tem mais de 40 anos. Segundo Valente e Almeida (1997), a área de pesquisa nasceu no início dos anos 70 a partir de algumas experiências na UFRJ, UFRGS e UNICAMP. Nos anos 80 se estabeleceu através de diversas atividades que permitiram que essa área hoje tenha uma identidade própria, raízes sólidas e relativa maturidade. A área passou a possuir cada vez mais pesquisadores interessados e eventos dedicados às questões que rodeiam o tema. No Brasil um dos principais eventos da área é o Simpósio Brasileiro de Informática na Educação (SBIE).

Desde a primeira edição realizada no Rio de Janeiro em 1990, o Simpósio Brasileiro de Informática na Educação se consolidou como o principal evento científico da área de informática aplicada à educação no Brasil. Para pesquisadores e educadores, o SBIE tem sido um importante ambiente de publicação de resultados, discussão de temas e tendências da área e interação entre grupos que estudam os diversos tópicos que envolvem a informática na educação. Além disso, o evento proporciona um ambiente para a troca de experiências e ideias com profissionais, professores, estudantes e 
pesquisadores nacionais e estrangeiros, estimulando ao surgimento e consolidação de grupos de pesquisa.

Esta pesquisa tem o propósito de realizar um mapeamento sistemático dos artigos publicados nas 12 últimas edições do SBIE (2001 - 2012), a fim de responder a seguinte pergunta de pesquisa. RQ: Como se caracteriza a pesquisa em informática na educação no Brasil? Nosso principal objetivo é consolidar informações quantitativas e qualitativas sobre a produção científica apresentada no SBIE de forma a contribuir com o avanço as pesquisas na Informática na Educação no país.

$\mathrm{O}$ artigo segue organizado em seis seções, a partir desta introdução. A segunda seção apresenta informações conceituais acerca da temática. A terceira seção relata experiências anteriores na mesma linha de pesquisa. Na seção subsequente são apresentados os procedimentos metodológicos de pesquisa adotados para realização dos trabalhos. Logo após, na quinta seção, os resultados do estudo são apresentados e discutidos e, por fim, na sexta seção são feitas as considerações finais.

\section{Estudos Secundários}

Os estudos primários podem ser caracterizados como pesquisas que são guiadas pelas hipóteses que se deseja averiguar ou compreender. Estes estudos são implementados quando há a necessidade de caracterizar uma determinada tecnologia em uso dentro de um contexto específico (BIOLCHINI et al., 2005). A literatura define alguns tipos de estudos primários que podem ser aplicados em pesquisas científicas. Os estudos de caso, os experimentos, os surveys, a pesquisa-ação e a etnografia são exemplos de métodos empíricos comumente aplicados em pesquisas primárias de diversas áreas, inclusive em Computação (MAFRA; TRAVASSOS, 2006; EASTERBROOK et al., 2011).

Por outro lado, os estudos secundários são aplicados para integrar os resultados oriundos de diversos estudos primários relacionados a um determinado tema. Este tipo de investigação bastante útil na identificação de evidências e na construção de conhecimento. $\mathrm{Na}$ engenharia de software, Kitchenham et al. (2004) adaptaram o método de revisões sistemáticas utilizado na medicina e nas ciências sociais para guiar a construção de revisões em diversos tópicos da engenharia de software (CAVALCANTI; da SILVA, 2011).

As revisões sistemáticas são o tipo mais comum de estudo secundário. Referemse a um método de pesquisa que visa obter e avaliar um conjunto de evidências pertencentes a um contexto específico, com a finalidade de integrar resultados experimentais e a sua aplicação poderá enfatizar a descoberta de problemas gerais e incentivar o diagnóstico e a análise de inconsistências encontradas ao comparar estudos individuais. As revisões sistemáticas convencionais agregam resultados sobre uma questão específica aplicada para resolver questões de pesquisa do tipo relacional e comparativa. Os mapeamentos sistemáticos são um tipo particular de revisão sistemática com uma visão mais ampla dos estudos primários e busca responder questões descritivas sobre tópicos específicos (MIAN et al., 2005; da SILVA et al., 2011).

\section{Trabalhos Relacionados}

Em 2011, Cavalcanti e da Silva desenvolveram e publicaram um estudo cujo objetivo foi fornecer uma análise abrangente e sistemática dos trabalhos científicos publicados 
no Simpósio Brasileiro de Engenharia de Software (SBES). Para tal, utilizaram uma metodologia de revisão sistemática de literatura visando extrair, catalogar, analisar e sintetizar dados de todos os artigos publicados em todas as 24 edições da SBES, em relação aos aspectos históricos, conceituais e metodológicos.

Da mesma forma, algumas pesquisas mapearam cenários mais específicos dentro da Informática na Educação. Em 1999 Fernandes e Santos mostraram os perfis dos grupos atuantes na área para que a comunidade de Informática na Educação na época pudesse compartilhar e disseminar trabalhos e projetos desenvolvidos. Em 2011 Bogoni e Pinho realizaram uma revisão sistemática sobre a utilização de simuladores baseados em Realidade Virtual no treinamento dos estudantes. Outras pesquisas deste tipo podem ser encontradas nos anais do SBIE. Freitas Jr et al. (2012) analisaram as publicações internacionais sobre métodos de engenharia do conhecimento como suporte aos processos de aprendizagem organizacional. Aureliano e Tedesco (2012) investigaram nas publicações do SBIE e do WIE processos de ensino-aprendizagem de programação para iniciantes. Neste estudo pretende-se desenvolver um processo semelhante ao apresentado por Cavalcanti e da Silva (2011), aplicando a técnica de pesquisa secundária (o mapeamento sistemático) para realizar uma análise abrangente e sistemática da pesquisa em informática na educação do Brasil através do seu principal simpósio, o SBIE.

\section{Procedimentos Metodológicos}

Em seu trabalho, Cavalcanti e da Silva (2011) discutem a taxonomia de Cooper (1984) através da qual foi sistematizado o método de revisões integrativas de literatura considerando cinco características: foco, objetivo, perspectiva, cobertura, organização e audiência. Assim como no trabalho relacionado (CAVALCANTI; da SILVA, 2011) esta pesquisa também utilizará a taxonomia de Cooper, com foco no método de pesquisa e com objetivo de identificar tendências nas publicações do SBIE. A cobertura desse estudo é exaustiva, sendo analisados todos os trabalhos publicados entre 2001 e 2012, disponibilizados na página da Comissão Especial de Informática na Educação da SBC. A perspectiva da apresentação é neutra, a organização é histórica, conceitual e metodológica e a audiência é mista, constituída por pesquisadores e profissionais com interesse pela área.

O processo de mapeamento sistemático foi composto por quatro etapas principais, baseado no processo para mapeamento sistemático em engenharia de software aplicado por Petersen et al. (2008).

\section{Etapa 1 - Definição do Escopo do Mapeamento}

Todo o planejamento do processo, definição das questões de pesquisa, critérios de busca e composição da equipe foi definida nesta etapa. A equipe foi formada por dois estudantes de mestrado e dois professores doutores. Os dois alunos foram responsáveis por ler, analisar e classificar todas as publicações coletadas. Caso houvesse divergência de classificação e os dois alunos não entrassem em um consenso após uma discussão, essa questão seria discutida e resolvida sob a visão de um dos dois professores da equipe. Juntos os pesquisadores realizaram a análise e classificação dos artigos para responder as questões divididas em três facetas:

Aspectos Históricos: Como se deu a evolução histórica das publicações do SBIE? 
a) Como ocorreu a distribuição da quantidade de artigos publicados no SBIE?

b) Quais são as organizações que mais participaram com publicações no SBIE?

c) Qual a quantidade de artigos escritos em inglês no SBIE?

Aspectos Conceituais: Quais os tópicos da informática na educação foram mais pesquisados no SBIE?

Para responder esta questão utilizou-se uma lista de tópicos de interesse em pesquisas de informática na educação construída a partir das listas disponíveis nos sites ainda ativos das edições do SBIE. Assim, uma lista com 23 tópicos de interesse da área foi construída através da chamada de trabalhos das edições de 2012, 2011, 2010, 2009, 2008, 2005, 2003 e 1999. As publicações coletadas puderam então ser distribuídas em 24 tópicos de pesquisa da Informática na Educação:

TABELA I - Tópicos de Interesse da Informática na Educação

\begin{tabular}{|c|c|}
\hline $\begin{array}{l}\text { 1. Adaptação, Personalização e e Avaliação } \\
\text { Aprendizagem Suportada por Computador. (APA) }\end{array}$ & 13. Inteligência Artificial Aplicada à Educação. (IAA) \\
\hline 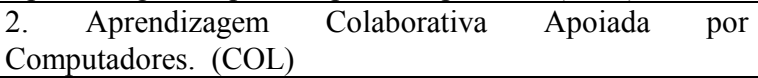 & $\begin{array}{l}\text { 14. Métodos, Processos e Ferramentas para Ensino de } \\
\text { Computação. (ENS) }\end{array}$ \\
\hline $\begin{array}{l}\text { 3. Arquiteturas e Métodos de construção de Software } \\
\text { Educativo. (ARQ) }\end{array}$ & $\begin{array}{l}\text { 15. Engenharia de Software Aplicada à Informática na } \\
\text { Educação. (ENG) }\end{array}$ \\
\hline 4. Aspectos Sociais da Informática na Educação. (SOC) & $\begin{array}{l}\text { 16. Mineração de Dados, Padrões e Repositórios Digitais de } \\
\text { Materiais Educacionais. (MIN) }\end{array}$ \\
\hline $\begin{array}{l}\text { 5. Avaliação e Desenvolvimento de Software Educativo. } \\
\text { (ADS) }\end{array}$ & 17. Objetos de Aprendizagem (OAP) \\
\hline 6. Comunidades Virtuais de Aprendizagem. (COM) & 18. Políticas para Informática na Educação. (POL) \\
\hline 7. Educação à Distância. (EAD) & 19. Realidade Virtual e aumentada na Educação. (REA) \\
\hline $\begin{array}{lllll}\text { 8. Fundamentos Pedagógicos } & \text { e } & \text { Psicológicos } & \text { da } \\
\text { Informática na Educação. (PED) } & & & \\
\end{array}$ & 20. Redes Sociais na Educação. (RED) \\
\hline 9. Hipermídia. (HIP) & 21. Simuladores e Jogos Educativos (JOG) \\
\hline 10. Interfaces de Software Educacional. (IHC) & 22. Tecnologias Wireless, Móvel e Ubíqua na IE. (UBI) \\
\hline 11. Informática na Educação Especial. (ESP) & 23. Usabilidade e Acessibilidade. (USA) \\
\hline 12. Informática na Escola e na Sala de Aula. (ESC) & 24. Web Semântica e Ontologias na Educação (ONT) \\
\hline
\end{tabular}

Aspectos Metodológicos: Quais os métodos empíricos de pesquisa utilizados pelos pesquisadores do SBIE?

\section{Etapa 2 - Busca por Estudos Primários}

A busca ocorreu de forma manual através site da Comissão Especial de Informática na Educação da SBC, no qual foram extraídos os artigos completos (full papers) dos anos de 2001 a 2012. Nesta etapa, artigos disponibilizados que só apresentavam o resumo da pesquisa e trabalhos cuja identificação dos autores não esteve disponível do corpo do texto foram excluídos do processo de mapeamento.

\section{Etapa 3 - Extração de dados}

Nesta fase buscou-se obter informações de cada um dos artigos encontrados, de forma a responder as perguntas de pesquisa. Para auxiliar este processo, o MS Excel foi utilizado.

\section{Etapa 4 - Análise e Síntese}

A partir dos dados extraídos na fase anterior deu-se o processo de interpretação dos resultados, criação de tabelas e gráficos para apresentação da informação e descrição das evidências identificadas nos estudos primários. 


\section{Resultados e Discussões}

Esta seção tem o intuito de apresentar uma análise descritiva sobre as publicações do SBIE nos últimos 12 anos, além de observações feitas ao longo da síntese dos dados coletados.

\section{A. Aspectos Históricos}

Este ponto discute a evolução das publicações sobre três aspectos: a) a distribuição de artigos publicados no evento; b) organizações mais ativas no SBIE e; c) o percentual dos artigos escritos em inglês publicados ao longo dos anos.

Como ocorreu a distribuição da quantidade de artigos publicados no SBIE?

A quantidade de artigos publicados no SBIE ao longo do período analisado, apesar de algumas baixas em certos momentos, foi crescente, principalmente a partir de 2007, como apresentado na figura 1. Foram publicados 835 trabalhos completos no simpósio. Isso demonstra o aumento da comunidade científica da área da Informática na Educação e o crescimento dos pesquisadores brasileiros pelo tema.

Figura 1. Evolução das publicações do SBIE ao longo dos anos

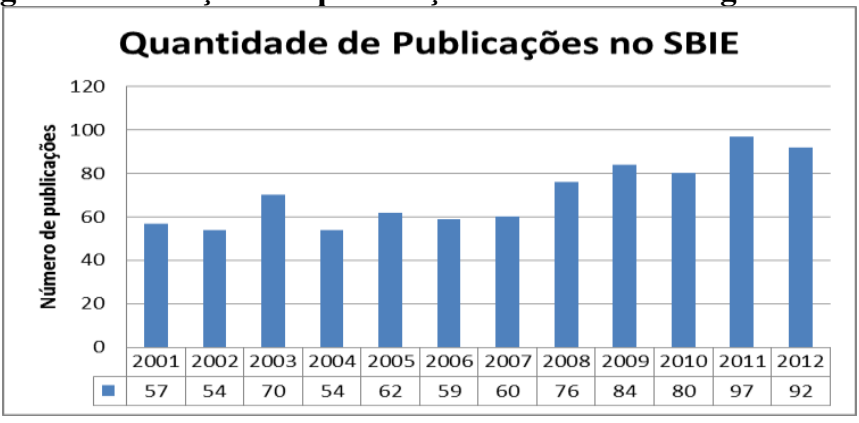

Quais são as organizações que mais participaram com publicações no SBIE?

Aproximadamente 250 instituições diferentes publicaram trabalhos no SBIE no período analisado, no qual foi possível observar a participação não somente de universidades públicas e privadas, mas também de empresas de desenvolvimento de software, ONGs e associações, além de órgãos públicos como prefeituras. Este dado mostra a importância do simpósio para a área e o nível de penetração dos resultados publicados no evento dentro da sociedade. A figura 2 apresenta a quantidade de instituições que publicaram no SBIE em cada ano.

Participaram do SBIE universidades das 5 regiões do país, além de alguns autores de instituições internacionais como Estados Unidos, Argentina, Holanda, França e Japão. Isto constata a importância do fórum e das pesquisas na área para resolver questões dos mais diversos tipos e em diferentes contextos sociais e econômicos, além do estímulo ao surgimento e consolidação de grupos de pesquisa.

A lista das dez organizações mais ativas em publicações no evento pode ser observada através da figura 3. A UFRGS foi a instituição que mais publicou trabalho com 138 artigos, cerca de 16,5\% do total (138/835), seguida da UFES com 7,1\% dos artigos publicados no evento (59/835). Na lista ainda é possível identificar a UNISINOS (5,7\% - 48/835), UFRJ (5,1\% - 43/835), USP (4\% - 33/835), UFC (5,8\% - 33/835), 
UFPE (3,7\% - 32/835), ITA (3,6\% - 30/835), UNICAMP e PUC-RS com 3,2\% (27/835) dos trabalhos, cada uma.

Figura 2. Quantidade anual de Instituições no SBIE

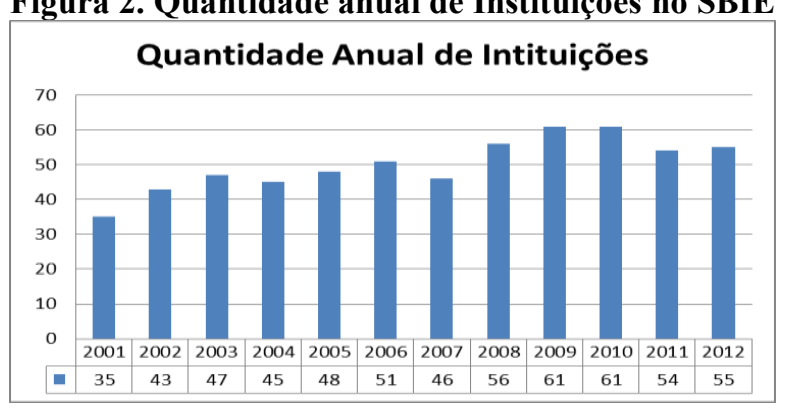

Figura 3. Organizações mais ativas no SBIE

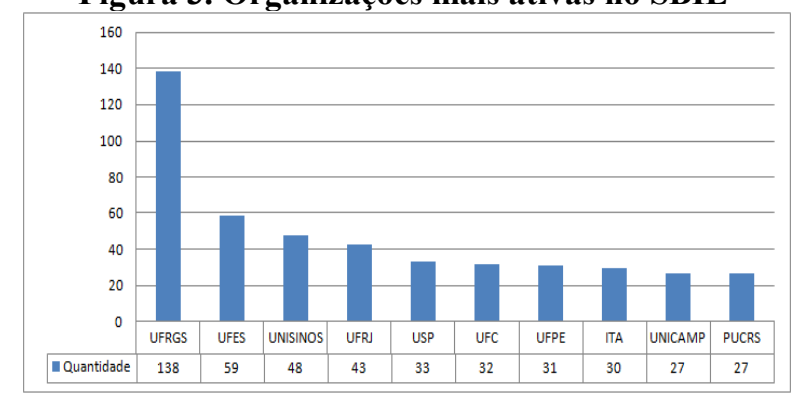

Qual a quantidade de artigos escritos em inglês no SBIE?

Neste ponto torna-se importante discutir que como observado por Cavalcanti e da Silva (2011), a existência de publicações no evento em inglês pode permitir a participação de revisões de outros países, contribuindo para o crescimento e divulgação do evento. Além disso, passa a existir a possibilidade de formação de grupos de pesquisa com cooperação internacional e resultados mais amplamente discutidos. No caso particular do SBIE pode-se inferir que as publicações de artigos em inglês têm seguido uma frequência relativamente baixa se comparada a outros eventos nacionais de grande porte como o Simpósio Brasileiro de Engenharia de Software, por exemplo. A figura 4 mostra que no período analisado, apenas 4,6\% (38/835) foram publicados em inglês.

Figura 4. Evolução das publicações do SBIE em Inglês

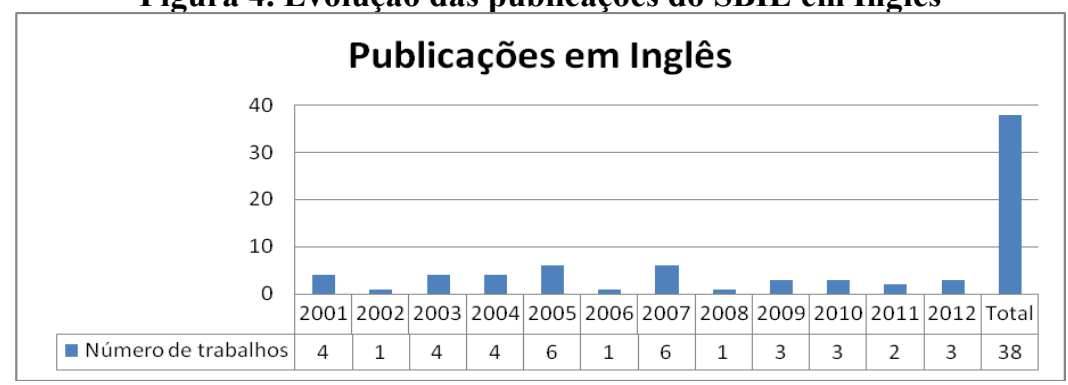

\section{B. Aspectos Conceituais}

Esta seção tem o objetivo de apresentar a distribuição das publicações analisadas nos tópicos de pesquisa da Informática na Educação, levando em consideração a lista de tópicos de interesse da TABELA I.

Quais os tópicos da informática na educação foram mais pesquisados no SBIE?

O principal interesse dos pesquisadores do SBIE é a publicação dos resultados da implementação de soluções computacionais aplicadas à educação. Neste sentido, Avaliação e Desenvolvimento de Software Educativo (ADS) é o tópico mais explorado na área, cobrindo $28,9 \%(241 / 835)$ do total de artigos publicados no período. Foi observado também que este é o tópico que mais se relaciona com os outros tópicos de 
interesse da lista, sendo que durante este período foram apresentados diversos tipos de software educativo para resolver os mais variados tipos de problemas em muitos contextos e para muitos públicos.

O segundo tópico mais pesquisado do SBIE foi a Educação à Distância (EAD) e os processos que envolvem esse tema. Foram 19,3\% dos trabalhos (161/835) voltados para esta questão, podendo-se destacar a implementação de solução para facilitar os trabalhos nos ambientes da internet que suportam o desenvolvimento das atividades, a proposta de arquiteturas e ferramentas de avaliação e o apoio ao aprendiz nas tarefas efetuadas à distância. Ao logo dos anos a evolução das pesquisas neste tema permitiram que este tópico estivesse relacionado com grande parte dos outros tópicos de interesse da área pela sua ligação com diversos contextos e problemas ainda carentes de solução.

Em terceiro lugar observou-se a forte exploração dos conceitos e ferramentas da Inteligência Artificial (IAA) em pesquisas da área, na qual 17,1\% (143/835) das pesquisas trabalharam direto ou indiretamente com este tópico. Este é um tema comumente usado para melhorar os processos desenvolvidos através do software educativo, provendo inclusive maior personalização e adaptação dos aprendizes em determinados contextos. Este é um tópico bastante ligado também ao processo de avaliação automática de estudantes e a inclusão de características afetivas e motivacionais ao software educativo.

A colaboração (COL) foi o quarto tópico mais pesquisado no SBIE com 13,3\% dos trabalhos (111/835). A abordagem colaborativa foi aplicada em diversas situações como uma forma de melhorar a experiência do usuário de software educativo. Mais do que isso, durante este período foram desenvolvidos diversos sistemas colaborativos no contexto da informática na educação visando as interações em rede como forma de enriquecer o pensamento individual e o aprendizado.

A figura 5 apresenta a distribuição dos trabalhos em todos os 24 tópicos de interesse definidos neste artigo. Uma discussão completa sobre cada tópico não pode ser realizada neste momento, por questões de espaço.

Figura 5. Tópicos da Área Explorados no SBIE

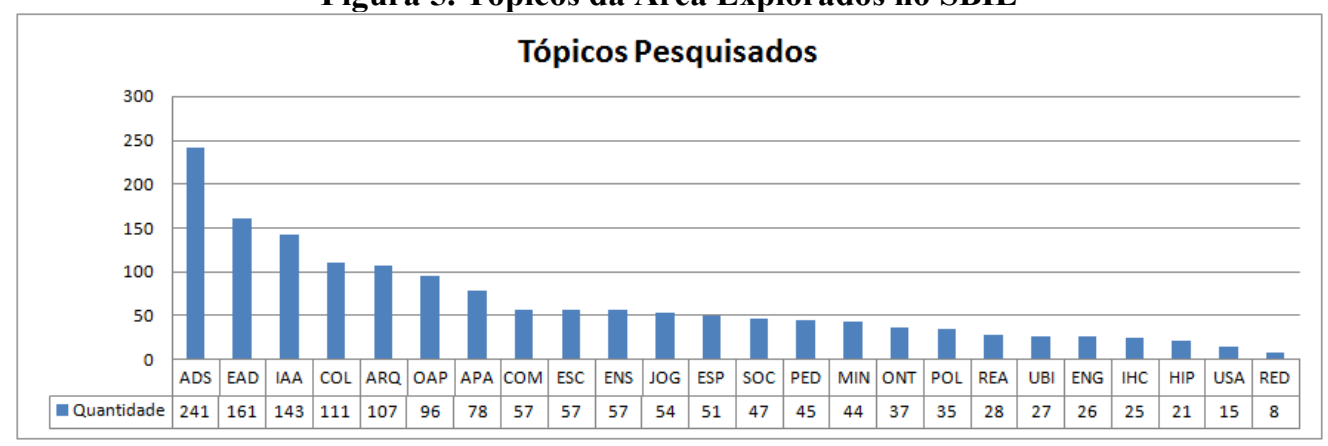

\section{C.Aspectos Metodológicos}

Este ponto do trabalho discute a distribuição das publicações por método de pesquisa empregado.

Quais os métodos de pesquisa utilizados pelos pesquisadores do SBIE? 
Um número muito elevado de artigos publicados no SBIE, no período analisado, não utilizou nenhum método empírico na pesquisa realizada. Mesmo apresentado boas propostas, principalmente de software educativo e arquiteturas para o desenvolvimento desses sistemas, em muitos casos os autores não realizaram nenhum tipo de validação dos resultados obtidos. A figura 6 ilustra a distribuição dos métodos de pesquisa por ano e a figura 7 mostra que ao passar dos anos cada vez mais pesquisadores passaram a utilizar métodos empíricos em suas pesquisas, porém esse número ainda é pequeno em relação à quantidade de trabalhos publicados por evento.

Apesar de apresentar uma linha de aumento inconstante, a utilização dos estudos de caso na área manteve um nível de crescimento considerável nos últimos anos. Este método representa $18,3 \%$ do total de artigos publicados no SBIE (153/835). Os experimentos formais (e quasi-experimentos) também apresentaram crescimento ao longo dos anos nas pesquisas da área, aparecendo em 8,7\% das pesquisas (73/835). A sequência segue com métodos pouco aplicados, os surveys com $1 \%(8 / 835)$, a pesquisaação com $0,7 \%(6 / 835)$ e por fim as etnografias em apenas $0,2 \%$ dos artigos $(2 / 835)$.

Figura 6. Métodos de pesquisa aplicados nos trabalhos do SBIE

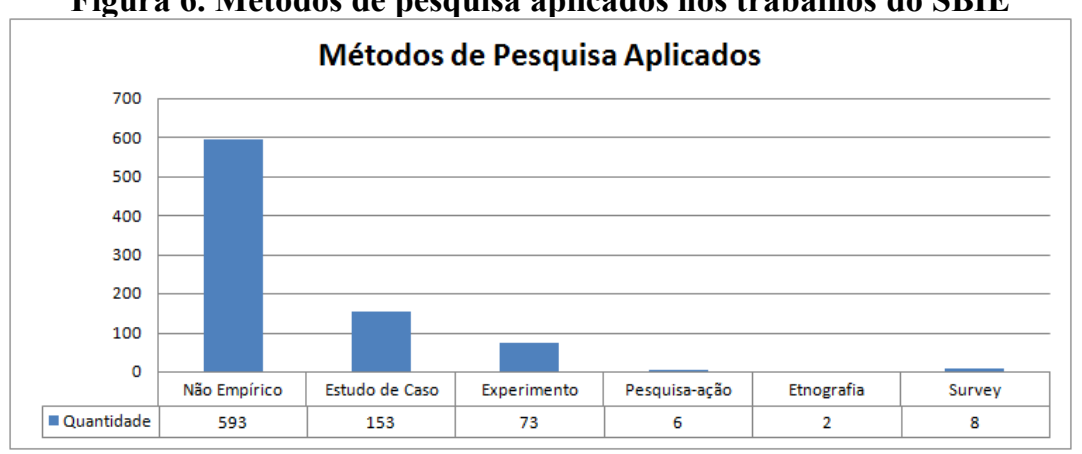

Figura 7. Distribuição dos métodos de pesquisa aplicados por ano

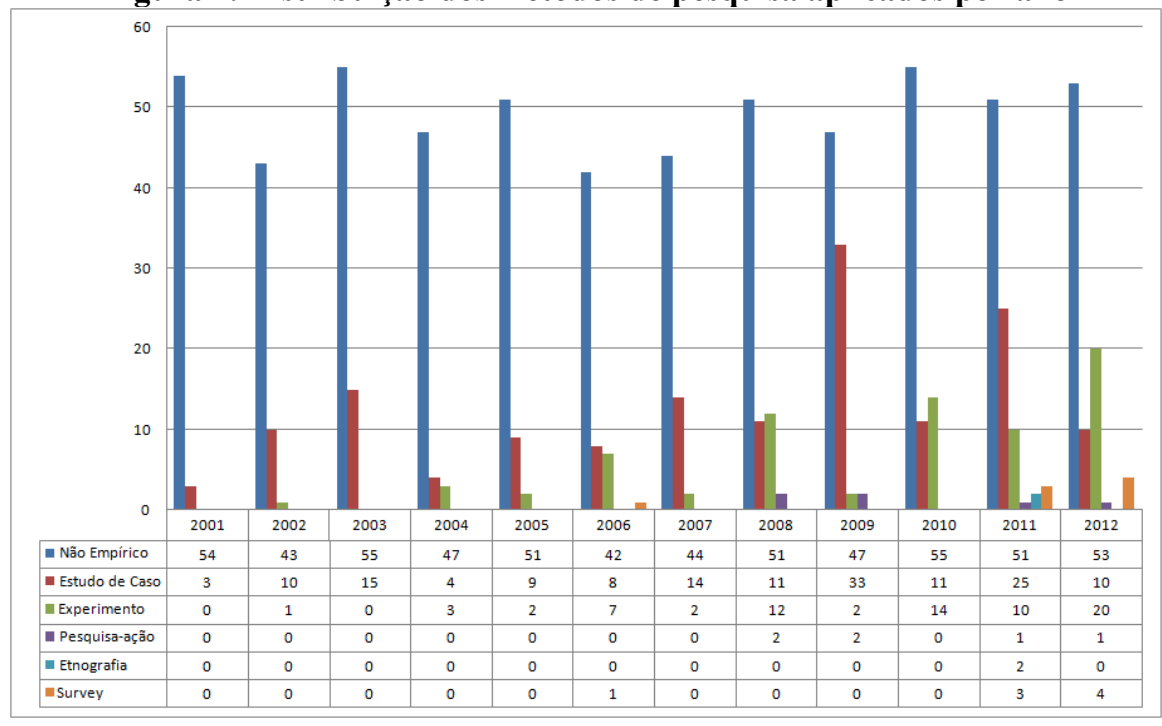

\section{Conclusões}

Através da análise de 835 trabalhos publicados no SBIE, principal evento em Informática na Educação do Brasil, pode-se concluir que no país a pesquisa nesta área é 
bastante diversificada em relação aos tópicos pesquisados, abrangendo diversos contextos, situações e público.

Durante o período coberto pelo mapeamento, observou-se que diferentes questões foram abordadas pelas pesquisas, não só na aplicação de ferramentas educacionais, como também nas propostas de políticas e relatos do uso do computador no meio educativo. Além disso, percebeu-se que enquanto alguns tópicos de pesquisa possuem grande representatividade outros possuem poucas pesquisas desenvolvidas, como é o caso da aplicação de redes sociais na educação e da usabilidade e acessibilidade do software educativo.

Os estudos primários analisados são uma importante fonte de informações sobre as diversas mudanças passadas pela área ao longo do tempo. Uma das evoluções mais importantes percebidas foi a aplicação de métodos empíricos como meio de avaliação dos resultados obtidos pelas das ferramentas desenvolvidas e métodos e processos propostos. Enquanto em 2001 somente o estudo de caso foi aplicado como método de pesquisa e em apenas três artigos, em 2009, por exemplo, o método foi usado em mais de um terço dos trabalhos. Além disso, em 2011, cinco métodos de pesquisa empírica foram identificados em pelo menos um artigo.

As publicações classificadas aqui como não empíricas representam as pesquisas de caráter fundamentalmente descritivo, como a simples apresentação de proposta de ferramentas, descrição de métodos e relato de atividades. Estas representarem mais da metade das publicações do evento, o que demonstra que a pesquisa em Informática na Educação no país ainda está se desenvolvendo em publicações de caráter avaliativo. É importante perceber que as pesquisas de caráter avaliativo demonstram certo nível de amadurecimento da área, aumento da confiabilidade dos resultados apresentados e permite o direcionamento apropriado das pesquisas e das discussões sobre o tema, tanto no meio acadêmico quanto no prático.

Por questões de espaço, outras conclusões realizadas sobre os dados coletados não puderam ser apresentadas neste artigo. A relação direta entre os tópicos de interesse presentes nas publicações do SBIE ou a distribuição destes tópicos por ano são exemplos de análises realizadas, porém não apresentadas. Uma versão ampliada deste artigo poderia apresentar estes resultados. Além disso, define-se como trabalhos futuros a identificação e análise dos trabalhos publicados no SBIE nos anos anteriores a $2001 \mathrm{e}$ que não estão disponíveis na página criada pela Comissão Especial de Informática na Educação da SBC.

\section{Agradecimentos}

O Professor Fabio Q. B. da Silva é bolsista de Desenvolvimento Tecnológico e Extensão Inovadora (DT) do CNPq, processo \#314523/2009-0. O Professor Alex Sandro Gomes é Bolsista DT Nível 2 no CNPq. Cleyton V. C. de Magalhães e Ronnie E. S. Santos são alunos de mestrado bolsistas da CAPES e da FACEPE, respectivamente. Gostaríamos de agradecer também ao Professor Herbert Lima e a equipe da UFC por ter compartilhado os arquivos dos artigos publicados no SBIE nos anos anteriores a 2001 e que servirão para a implementação dos trabalhos futuros dessa pesquisa. 


\section{Referências}

AURELIANO, V. C. O.; TEDESCO, P. C. A. R.. Ensino-aprendizagem de Programação para Iniciantes: uma Revisão Sistemática da Literatura focada no SBIE e WIE. Anais do Anais do $23^{\circ}$ Simpósio Brasileiro de Informática na Educação (SBIE 2012), ISSN 23166533. Rio de Janeiro, 2012.

BIOLCHINI, J.; MIAN, P. G.; NATALI, A. C. C.; TRAVASSOS, G. H. Systematic Review in Software Engineering. Relatório Técnico (Programa de Engenharia de Sistemas e Computação) Universidade Federal do Rio de Janeiro - UFRJ - Rio de Janeiro, 2005.

BOGONI, T. N.; PINHO, M.S. Uma Revisão Sistemática de Simuladores de Endodontia

Equipados com Dispositivos Hápticos. Anais do Anais do $22^{\circ}$ Simpósio Brasileiro de Informática na Educação (SBIE 2011), ISSN 2176-4301. Aracaju, 2011.

CAVAlCANTI, T. R; da SILVA, F. Q. B. Historical, Conceptual, and Methodological Aspects of the Publications of the Brazilian Symposium on Software Engineering: A Systematic Mapping Study. Anais do 25th Brazilian Symposium on Software Engineering (SBES), São Paulo, 2011.

Comissão Especial de Informática na Educação da SBC. Anais do SBIE. Acesso em maio de 2012. Disponível em < http://www.br-ie.org/index.php/anaissbie $>$.

COOPER, H.M. The integrative research review: A systematic approach. Applied social research methods series. Beverly Hills, v.2, 1984.

da SILVA, F. Q. B.; SANTOS, A. L. M.; SOARES, S. C. B.; FRANÇA, A. C. C.; MONTEIRO, C. V. F.; MACIEL, F. F. Six years of systematic literature reviews in software engineering: An updated tertiary study. Information and Software Technology, 2011.

EASTERBROOK, S.; SINGER, J.; STOREY, M.; DAMIAN, D. Selecting Empirical Methods for Software Engineering Research. Computer Science Department, University of Toronto, 2011.

FERNANDES, C. T.; SANTOS, N. Pesquisa e Desenvolvimento em Informática na Educação no Brasil - Parte I. Revista Brasileira de Informática na Educação. Número 4, 1999.

FREITAS JR, V.; ANDERLE, D. F.; SANTOS, J. L. S.; STEIL, A. V. Engenharia do Conhecimento como suporte aos processos de Aprendizagem Organizacional. Anais do Anais do $23^{\circ}$ Simpósio Brasileiro de Informática na Educação (SBIE 2012), ISSN 23166533. Rio de Janeiro, 2012.

KITCHENHAM, B.; DYBÅ, T.; JØRGENSEN, M. Evidence-based Software Engineering. 26th International Conference on Software Engineering, (ICSE '04), Proceedings. IEEE, Washington DC, USA, pp 273 - 281, 2004.

MAFRA, S. N.; TRAVASSOS, G. H. Estudos Primários e Secundários Apoiando a Busca por Evidência em Engenharia de Software. Relatório Técnico (Programa de Engenharia de Sistemas e Computação) Universidade Federal do Rio de Janeiro - UFRJ - Rio de Janeiro, 2006.

MIAN, P.; CONTE, T.; NATALI, A.; BIOLChINI, J.; MENDES, E.; TRAVASSOS, G. H. Lessons Learned On Applying Systematic Reviews To Software Engineering. 3rd International Workshop "Guidelines For Empirical Work" In the Workshop Series On Empirical Software Engineering (Wsese). Proceedings, 2005.

PETERSEN, K.; FELDT, R.; MUJTABA, S.; MATTSSON, M. Systematic Mapping Studies in Software Engineering. 12th International Conference on Evaluation and Assessment in Software Engineering (EASE). University of Bari, Italy, 26 - 27 June 2008. 